\title{
A cost-effective, community-based, mosquito- trapping scheme that captures spatial and temporal heterogeneities of malaria transmission in rural Zambia
}

Chadwick H Sikaala ${ }^{1,2^{*}}$, Dingani Chinula ${ }^{1}$, Javan Chanda', Busiku Hamainza ${ }^{1,2}$, Mulenga Mwenda$^{1}$, Isabel Mukali ${ }^{1}$, Mulakwa Kamuliwo', Neil F Lobo ${ }^{3}$, Aklilu Seyoum² $^{2}$ and Gerry F Killeen ${ }^{2,4}$

\begin{abstract}
Background: Monitoring mosquito population dynamics is essential to guide selection and evaluation of malaria vector control interventions but is typically implemented by mobile, centrally-managed teams who can only visit a limited number of locations frequently enough to capture longitudinal trends. Community-based (CB) mosquito trapping schemes for parallel, continuous monitoring of multiple locations are therefore required that are practical, affordable, effective, and reliable.

Methods: A CB surveillance scheme, with a monthly sampling and reporting cycle for capturing malaria vectors, using Centers for Disease Control and Prevention light traps (LT) and Ifakara Tent Traps (ITT), were conducted by trained community health workers (CHW) in 14 clusters of households immediately surrounding health facilities in rural south-east Zambia. At the end of the study, a controlled quality assurance (QA) survey was conducted by a centrally supervised expert team using human landing catch (HLC), LT and ITT to evaluate accuracy of the CB trapping data. Active surveillance of malaria parasite infection rates amongst humans was conducted by CHWs in the same clusters to determine the epidemiological relevance of these $C B$ entomological surveys.
\end{abstract}

Results: CB-LT and CB-ITT exhibited relative sampling efficiencies of 50 and 7\% respectively, compared with QA surveys using the same traps. However, cost per sampling night was lowest for CB-LT (\$13.6), followed closely by CB-ITT (\$18.0), both of which were far less expensive than any QA survey (HLC: \$138, LT: \$289, ITT: \$269). Cost per specimen of Anopheles funestus captured was lowest for CB-LT (\$5.3), followed by potentially hazardous QA-HLC (\$10.5) and then CB-ITT (\$28.0), all of which were far more cost-effective than QA-LT (\$141) and QA-ITT (\$168). Time-trends of malaria diagnostic positivity (DP) followed those of An. funestus density with a one-month lag and the wide range of mean DP across clusters was closely associated with mean densities of An. funestus caught by CB-LT $(P<0.001)$.

Conclusions: CB trapping schemes appear to be far more affordable, epidemiologically relevant and cost-effective than centrally supervised trapping schemes and may well be applicable to enhance intervention trials and even enable routine programmatic monitoring of vector population dynamics on unprecedented national scales.

Keywords: Community-based, Trapping scheme, Cost-effective, Anopheles funestus

\footnotetext{
* Correspondence: chadsikaala@gmail.com

${ }^{1}$ National Malaria Control Centre, Chainama Hospital College Grounds, Off

Great East road, P.O. Box 32509 Lusaka, Zambia

${ }^{2}$ Vector Biology Department, Liverpool School of Tropical Medicine,

Pembroke Place, Liverpool L3 5QA, UK

Full list of author information is available at the end of the article
} 


\section{Background}

Despite the impressive successes of long-lasting insecticidal nets (LLINs) and indoor residual spraying (IRS), which selectively target malaria vectors when they feed or rest inside human habitations, these front line vector control tools have rarely achieved complete elimination of malaria outside of areas that had marginal transmission levels to begin with [1-3]. These fundamental limits of what can be achieved with IRS or LLINs are primarily defined by the behavioural traits of mosquitoes [2,4-10], most of which appear to have always been present in these populations [2,4-7] so they are better described as preexisting behavioural resilience (Figure 1A) [6,7]. On the other hand, recent modelling analyses [11] have illustrated how apparently altered distributions of feeding times and locations following scale-up of LLIN or IRS cannot be

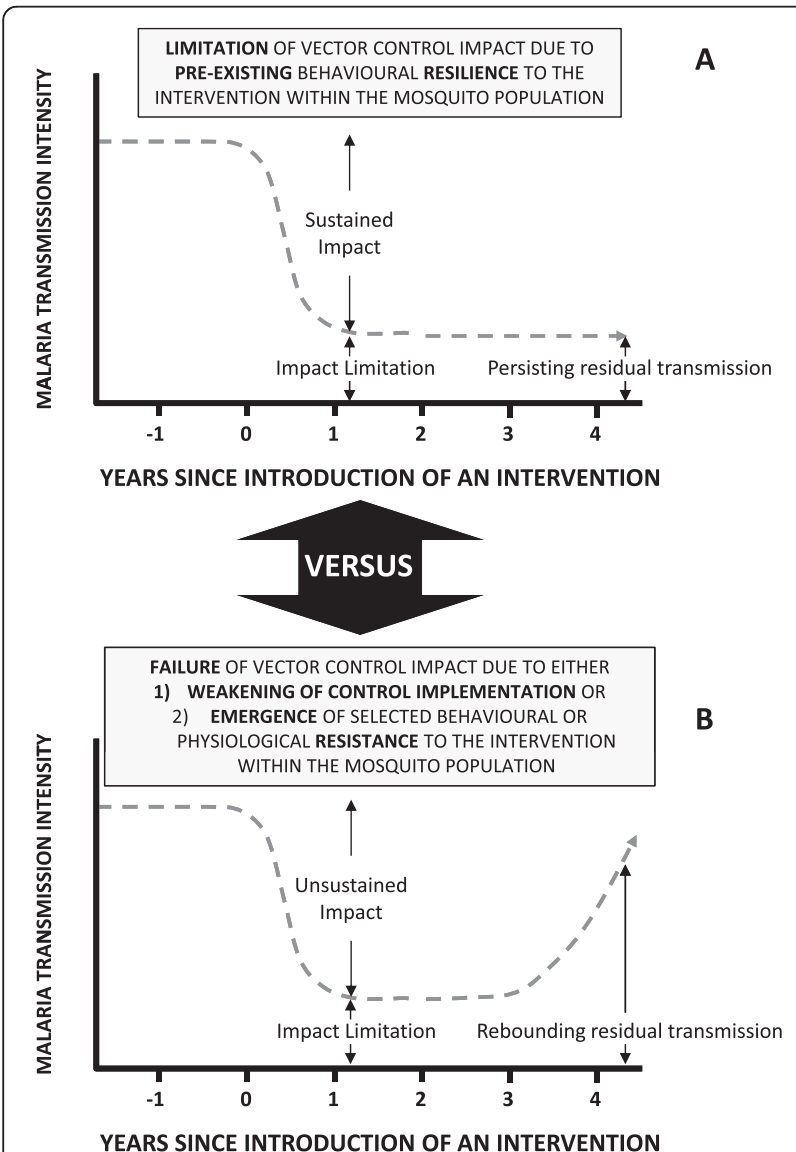

Figure $1 \mathrm{~A}$ schematic illustration of the differing trajectories of impact of an intervention upon malaria transmission by a vector population under the distinctive scenarios of either (A) stable limitation of sustained impact arising from expression of preexisting behavioural traits within a resilient vector population, or (B) failure of impact and resurgence of malaria transmission when, either intervention programme implementation quality and coverage weakens, or selected behavioural or physiological traits emerge within an increasingly resistant, rebounding vector population $[2,4,6,7,12]$. simply explained in terms of deferred feeding by hungry mosquitoes and may represent emergence of selected, heritable behavioural resistance in the strict sense (Figure 1B) $[6,7]$. Furthermore, resurgent malaria has been repeatedly associated with, not only failures of implementation and funding for vector control programmes, but also with emergence of physiological resistance to insecticides [12]. It is therefore crucial to distinguish between such fundamental limitations of a given vector control strategy, reflecting incomplete but nevertheless valuable levels of sustainable impact (Figure 1A), and a genuine failure of an intervention programme that results in rebounding vector populations and malaria transmission (Figure 1B).

The only way in which suppression or resurgence of malaria transmission can be unambiguously attributed to the success or failure of interventions to control responsible vectors will be to monitor their population dynamics longitudinally. Currently, across sub-Sahara Africa, almost all monitoring of vector populations is limited to detecting physiological resistance to prioritize optimal selection of active ingredients for intradomiciliary insecticidal-based interventions. It has therefore been suggested that robust longitudinal sentinel surveillance systems need to be established so that national malaria control programmes (NMCPs) can continually monitor physiological and behavioural traits, and assess their relevance to intervention selection, by evaluating their impact upon the population dynamics of target vector species $[6,13]$.

However, the cost of implementing adult mosquito surveillance through conventional teams of specialist entomologists may be prohibitive in impoverished African countries $[14,15]$. Conventional longitudinal entomological monitoring strategies rely operationally upon trained specialist technical staff managed centrally usually by academic or research institutions, so they are usually limited in both their geographic scope and the frequency of sampling at any survey location. The availability and cost of the expert human resources required to sustain such specialist teams is also limiting [14-16]. Mosquito species composition, abundance and transmission potential is not only altered by successful implementation of vector control measures $[2,6,8,13]$, it also varies dramatically geographically and seasonally. It is therefore difficult to envision how conventional, centralized entomological surveillance teams could capture such spatial and temporal patterns in a representative manner on national scales because they simply cannot reach all sentinel survey locations often enough to provide a robust representation of longitudinal trends at each one.

Decentralized systems that adapt cost-effective trapping methods to local, longitudinal application by resident community-based (CB) staff therefore represent an 
attractive alternative $[14,15]$. Implementation of CB trapping schemes presents two important challenges: 1) selection of traps, and protocols for their use, that are safe practical and convenient enough for $\mathrm{CB}$ staff to apply them reliably in the absence of daily supervision, and 2) independent quality assurance (QA) of this unsupervised surveillance process so that the accuracy and limitations of the derived data can be quantified as a prerequisite to critical interpretation. To date, however, only one CB mosquitotrapping scheme, designed to support a municipal-scale, larval source management programme in Dar es Salaam in Tanzania, has been critically evaluated through both QA of the derived entomological data and appraisal of its epidemiological relevance in terms of its ability to predict malaria infection risk among humans [15]. This first validated $\mathrm{CB}$ trapping scheme was also more sensitive, in terms of total numbers of mosquito caught, than the centrally supervised scheme used to conduct QA, because it was much more intensive and at the same time spatially extensive [15]. Furthermore, CB trapping results in Dar es Salaam were predictive of malaria risk infection amongst humans despite the fact that vector populations were remarkably sparse in this low transmission urban area [15]. However, the generalizability of this study to a wider variety of settings is not only limited by its local geographic scope, but also by the fact that it relied on entirely upon a locally designed Ifakara Tent Trap (ITT) $[17,18]$ because this was shown to be the only safe, sufficiently sensitive capture method in this context where Anopheles gambiae is the predominant species maintaining transmission [19].

Over the last decade, Zambia has made substantial progress toward implementing an ambitious strategic plan aiming to protect every at-risk individual in the country against malaria with either LLINs or IRS [20]. As insecticide resistance has now been clearly identified within the country, it is essential to develop a sustainable platform to monitor vector species composition, behaviour and transmission capacity on a national scale for the first time. Recent comparative evaluations of various mosquitotrapping methods, in rural south-east Zambia [21], where malaria transmission is primarily maintained by Anopheles funestus, demonstrated that the Centers for Disease Control and Prevention miniature Light Trap (LT) and ITT [18] both performed reasonably well as methods for capturing host-seeking mosquitoes and also suggested that they could be applied across a much larger geographic area through a more practical and scalable CB system. This manuscript describes an evaluation of the applicability of $\mathrm{CB}$ trapping schemes, using these two candidate capture methods, to assess their effectiveness for sampling malaria vectors across different times and locations, as well as their overall cost effectiveness and ability to predict human malaria infection risk in the same rural Zambian transmission system [21].

\section{Methods}

\section{Study area}

The study was conducted in Luangwa and Nyimba districts, located approximately $255 \mathrm{~km}$ and $325 \mathrm{~km}$, respectively, east of Lusaka, the capital city of Zambia (Figure 2). There are about 25,000 and 85,000 inhabitants in Luangwa and Nyimba, respectively, who predominantly practice seasonal farming, fishing and animal husbandry as their primary livelihood [22]. Malaria prevalence in this part of Zambia ranges from 9 to $22 \%$, with by far the lowest prevalence in the flat, sandy southern half of Luangwa [23]. Within this study area, intensive monitoring of malaria infection among the humans, and of human-biting mosquito densities in and around their houses, was carried out on a monthly survey cycle by resident community health workers (CHWs) in 14 population clusters centred around health facilities between January 2011 and March 2013 [22].

Between 2005 and 2012, Luangwa and Nyimba districts received repeated mass distributions of LLINs, complemented by routine distribution through antenatal clinics. As a result, 66 and 43\%, respectively of children under five years of age in Luangwa and Nyimba reported using a net the previous night by 2010 [24]. IRS was implemented between October and November 2010, using deltamethrin (K-Othrine WG ${ }^{\odot}$ 250, Bayer Environmental Science, South Africa) in the south of Luangwa district. At the same time of year in 2011, some of these villages in southern Luangwa district were sprayed with lambdacyhalothrin (Icon ${ }^{\oplus} 10$ Capsule Suspension (CS) formulation, Syngenta Crop Protection AG, Switzerland) while others, as well as several in Nyimba district, were sprayed with an emulsifiable concentrate (EC) formulation of the organophosphate pirimiphos-methyl (Actellic ${ }^{\odot}$ EC, Syngenta Crop Protection AG, Switzerland) to mitigate against the resistance to pyrethroids among $A n$. funestus populations in the area [25]. A year later 2012, the same regime was applied except that the selection of villages sprayed with pirimiphos methyl in Nymiba was changed and, in some of them, the EC formulation was replaced with a micro-encapsulated formulation of the same active ingredient (Actellic ${ }^{\circ}$ 300CS, Syngenta Crop Protection AG, South Africa).

\section{Longitudinal malaria parasite surveillance in the human population}

Fourteen population clusters of approximately 1,000 residents were selected in both districts (seven per district), each centred around a public sector health facility, in which each household was visited monthly by a CHW offering testing and treatment for malaria [22]. Three CHWs were recruited for this task, of which two enrolled approximately 60 households while the third, who was also responsible for $\mathrm{CB}$ mosquito trapping as described below, enrolled 45 households for parasitological 


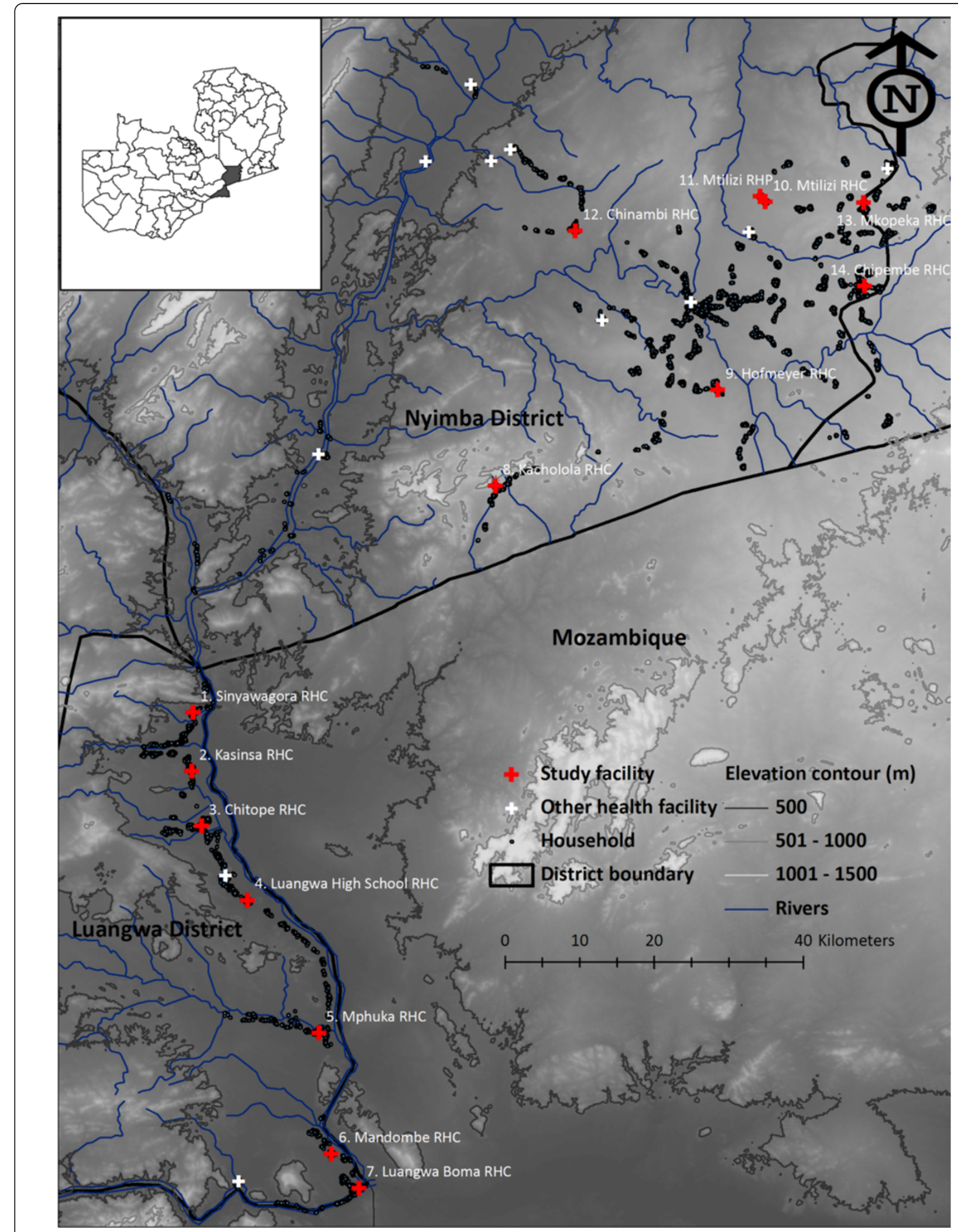

Figure $\mathbf{2}$ Location of study site, and numbered survey clusters around health facilities, in Zambia. 
surveillance. CHWs enlisted households in order of their proximity to the health facility and collected small fingerstick blood samples from all consenting and assenting household members who were present on a designated date each month for each household and tested on the spot using the MAL Pf Rapid Diagnostic Test (RDT) kit (ICT Diagnostic, Cape Town, South Africa) that detects histidine rich protein-2 (HRP-2) antigen. All individuals that tested positive in the field for the presence of malaria parasite antigen were provided with artemether-lumefantrine (Coartem, Norvatis Pharma AG, Basel, Switzerland) freeof-charge in accordance with the national guidelines. Between these active visits, individuals who felt sick or had symptoms were encouraged to seek medical care from their assigned CHW or the nearest health facility so cases were also detected passively. All the recruited CHWs were remunerated as casual labourers at a rate of ZMW 350 (\$66.9) per month. Mean diagnostic positivity rates of residents in individual clusters tested during monthly activity visits to their households ranged from 6 to $47 \%$ with an overall mean of $20.3 \%(11,851 / 58,500)$ across all age groups [22], approximately consistent with the recent National Malaria Indicator Survey [23] that describes a mean infection prevalence of $19.5 \%$ in cross sectional household surveys in the rural districts of Zambia.

\section{Community-based mosquito-trapping scheme}

In order to assess the effectiveness of $\mathrm{CB}$ surveillance of adult mosquito populations, one of the three CHWs in each cluster (specifically the ones with 45 households to survey) had additional training in basic entomology. One exception was at Luangwa High School (cluster 4), where two out of the three CHWs were engaged in conducting entomological surveillance of adult mosquito populations with one covering 45 while the other 60 households in the surveys of infection among the human residents. Fifteen houses per cluster for mosquito trapping were selected semi-arbitrarily to be well distributed across the cluster, with the exception of Luangwa High School where this figure was doubled to 30 due to the involvement of an additional CHW in mosquito trapping. Therefore, the targeted number of trapping nights per house per month was one. The cluster, village, and household codes, and household owner name for each household were recorded for all 299 households where $\mathrm{CB}$ surveys of mosquitoes were conducted. A consistent date of the month for mosquito trapping using the LT and ITT at each house was pre-agreed with each household head. The LTs were placed inside the house on the foot end of an occupied sleeping space already covered with LLIN at a height of approximately $1.5 \mathrm{~m}$ above the floor whilst an adult male from the same household occupied an ITT placed immediately outside, approximately $5 \mathrm{~m}$ away from the house where the LT was installed. The only occasion volunteers where replaced was in cases of illness, resignation or unreliability. Due to the inconvenience of the bulkiness of the ITT $[15,21]$ CHWs were provided with spare parts to maintain their bicycles to facilitate transport of the traps from one household to another during the study period. Mosquito traps were set up in the evenings and captured mosquitoes were collected by aspiration as early as was convenient the next morning. CHWs were trained to sort mosquitoes to genus level by eye, to store them over silica, and to keep it desiccated. Anopheles specimens were stored individually in $1.5 \mathrm{ml}$ microcentrifuge tubes while culicines were pooled in ziplock bags. Based on this crude morphological classification, the numbers of mosquitoes caught were recorded on a simple form by the CHW.

A team from the centralized National Malaria Control Centre (NMCC) entomological team collected the mosquito samples from all of the clusters once per month and delivered them to the central laboratory at the NMCC in Lusaka. At the central laboratory, anopheline mosquito samples were subjected to further morphological identification [26] and the data entered into an Excel sheet. Then the An. gambiae complex and $A n$. funestus group were taken to the molecular laboratory for further analysis and long-term storage. CB mosquito trapping was conducted continuously from January 2011 to April 2013 in Luangwa and from April 2011 to April 2013 in Nyimba district.

\section{Quality assurance surveys of the community-based trapping} In order to assess the validity of the $\mathrm{CB}$ trapping schemes using the LTs and ITTs, a QA team was assembled towards the end of the study. This team was recruited selectively from among the most experienced CHWs who were involved during the previous trap efficacy study in Chisobe village of Luangwa district. None of these team members had any other responsibilities within this particular study and were supervised by a technical team of trained entomologists from the central level at NMCC.

To validate the $\mathrm{CB}$ trapping schemes, the $\mathrm{QA}$ team visited the same households that the $\mathrm{CB}$ team had placed their traps a day or two earlier. The trapping efficacy of LT and ITT applied by the QA team, and their efficiency and effectiveness as applied by the CHWs, were compared with the gold standard human landing catches technique (HLC) [27] conducted by one male adult volunteer indoors and another outdoors. As described above, every month, on a date that was pre-agreed with the household owner, the CB team placed the LT indoors and ITT outdoors, and then at the next household on the schedule the following day. The QA team followed this sequence but delayed by a day or two to enable them have at least two houses to re-survey that the CHW had surveyed no more than three days previously. The QA team conducted HLC 
indoors and outdoors in one of the two houses while the other was surveyed with LT indoors and ITT outdoors. During this process a QA team member slept in the ITT. On the following day, the pair of participants conducting HLCs would remain in the same house but would apply the LT and ITT methods, while the other pair also stayed in the same house as the previous night but applied HLC. Therefore, each cluster was visited for at least one night by the QA team with a lag of only a day or two after $\mathrm{CB}$ catches in or around the same houses. The only exception was cluster 10, which the QA team never visited because the households were closely situated to those of cluster 11. Therefore only one of the two clusters was sampled for convenience. A specific form was used to record the data including the cluster name, village name, household code, household owner name, date, and trapping method. All trapping methods were applied by the QA teams between 19:00 and 07:00 hours. The CHWs were informed in advance about the QA team so that they could conveniently get consent from the household owners for the additional days of mosquito collections.

For QA surveys, samples of mosquitoes were collected and morphologically differentiated to genus level individually in the field. Female Anopheles mosquitoes were further separated, recorded and preserved individually in microcentrifuge tubes over desiccated silica gel. All males were recorded and discarded.

\section{Mosquito processing in the laboratory}

Further morphological identification of Anopheles to species group or complex [26] was conducted at the NMCC main laboratory. Female Anopheles samples were processed for detection of circumsporozoite protein ELISA [28], including confirmation following boiling of the head-thorax homogenates to prevent false-positive,[29] and polymerase chain reaction (PCR) identification of species within the $A n$. gambiae complex [30] or An. funestus group [31].

\section{Entomological and epidemiological data analysis}

Data were entered using Microsoft Excel 2007 and analysed using $\mathrm{R}$ statistical analysis software version 2.15.1, augmented with lattice, matrix and lme4 packages. To estimate the relative trapping efficiency of the different trapping schemes, a generalized linear mixed model (GLMM) was fitted using the number of mosquitoes of a given taxon as the Poisson-distributed dependent variable and trapping scheme as a categorical independent variable with five levels (CB-LT, CB-ITT, QA-HLC, QA-LT and QA-ITT). In order to account for spatial and temporal heterogeneity, as well as for over-dispersion, date, as well as households nested within clusters were treated as random effects. To ensure full comparability, data from the CB surveys collected more than seven days before or after a survey by the QA team in the same cluster were excluded from this analysis, so this comparison relates only to selected observations from the last three months of the study when both surveys were operational and overlapped in space and time.

Further, in estimating how An. funestus abundance predicts malaria infection risk among the human population, a GLMM was fitted with R statistical software augmented as above, with RDT results as the binomial dependent variable while the base 10 logarithm of the mean $A n$. funestus catch per LT for each cluster, estimated from the

Table 1 Total and unadjusted mean catches of malaria vectors and other mosquito species by community-based and quality assured sampling schemes

\begin{tabular}{|c|c|c|c|c|c|c|}
\hline \multirow[b]{2}{*}{ Trapping method: } & \multicolumn{4}{|c|}{ Quality assurance } & \multicolumn{2}{|c|}{ Community-based } \\
\hline & HLC indoor & HLC outdoor & LT & ITT & LT & ITT \\
\hline Person trap-nights & 20 & 20 & 20 & 20 & 3171 & 2195 \\
\hline Number of houses sampled & 20 & 20 & 20 & 20 & 505 & 432 \\
\hline Mean trap-nights per surveyed house & 1.0 & 1.0 & 1.0 & 1.0 & 6.3 & 5.1 \\
\hline Mean trap-nights per cluster & 1.5 & 1.5 & 1.5 & 1.5 & 226.6 & 156.8 \\
\hline \multicolumn{7}{|l|}{ Total catch of female mosquitoes } \\
\hline Anopheles funestus & 174 & 149 & 66 & 46 & 5,827 & 865 \\
\hline Anopheles quadriannulatus & 10 & 2 & 0 & 0 & 613 & 60 \\
\hline Other anophelines & 9 & 26 & 0 & 0 & 591 & 35 \\
\hline Culex species & 426 & 394 & 94 & 82 & 9,548 & 1,666 \\
\hline \multicolumn{7}{|l|}{ Mean catch of female mosquitoes } \\
\hline Anopheles funestus & 8.7 & 7.5 & 3.3 & 2.3 & 1.8 & 0.4 \\
\hline Anopheles quadriannulatus & 0.5 & 0.1 & 0.0 & 0.0 & 0.0 & 0.0 \\
\hline Other anophelines & 0.5 & 1.3 & 0.0 & 0.0 & 0.2 & 0.0 \\
\hline Culex species & 21.3 & 19.7 & 4.7 & 4.1 & 3.0 & 0.8 \\
\hline
\end{tabular}


Poisson model described above, was included as a continuous independent variable. Note, however, that to obtain specific estimates of the mean catches of An. funestus at each cluster, the model described above has to be modified so that cluster was treated as a categorical variable, rather than a random effect, and no intercept was included so that those estimates would be absolute rather than relative to an arbitrary reference group. Age categories of RDT-tested participants and date were treated as random effects and, to avoid any confounding effects household clustering would have on the An. funestus catch estimates, individual households were included nested within clusters as random effects. Data selected for this analysis of the dependence of malaria infection risk upon vector densities were restricted to the period from the onset of the study in January 2011 to September 2011 to avoid any confounding effects that the introduction of IRS in October and November 2011 would have on the densities or infection prevalence.

\section{Cost-effectiveness analysis}

This QA exercise was conducted for only the three final months of the study (February to April, 2013) in 13 of the 14 clusters, each of which was visited at least once using motorized transport provided to the QA team for that period. The government employed technical team members and the driver received their normal per diems during this period, which were ZMW500 (\$95.6) and ZMW300 (\$57.4) per night, respectively. The cost incurred also included vehicle fuel, maintenance and depreciation (purchase cost of

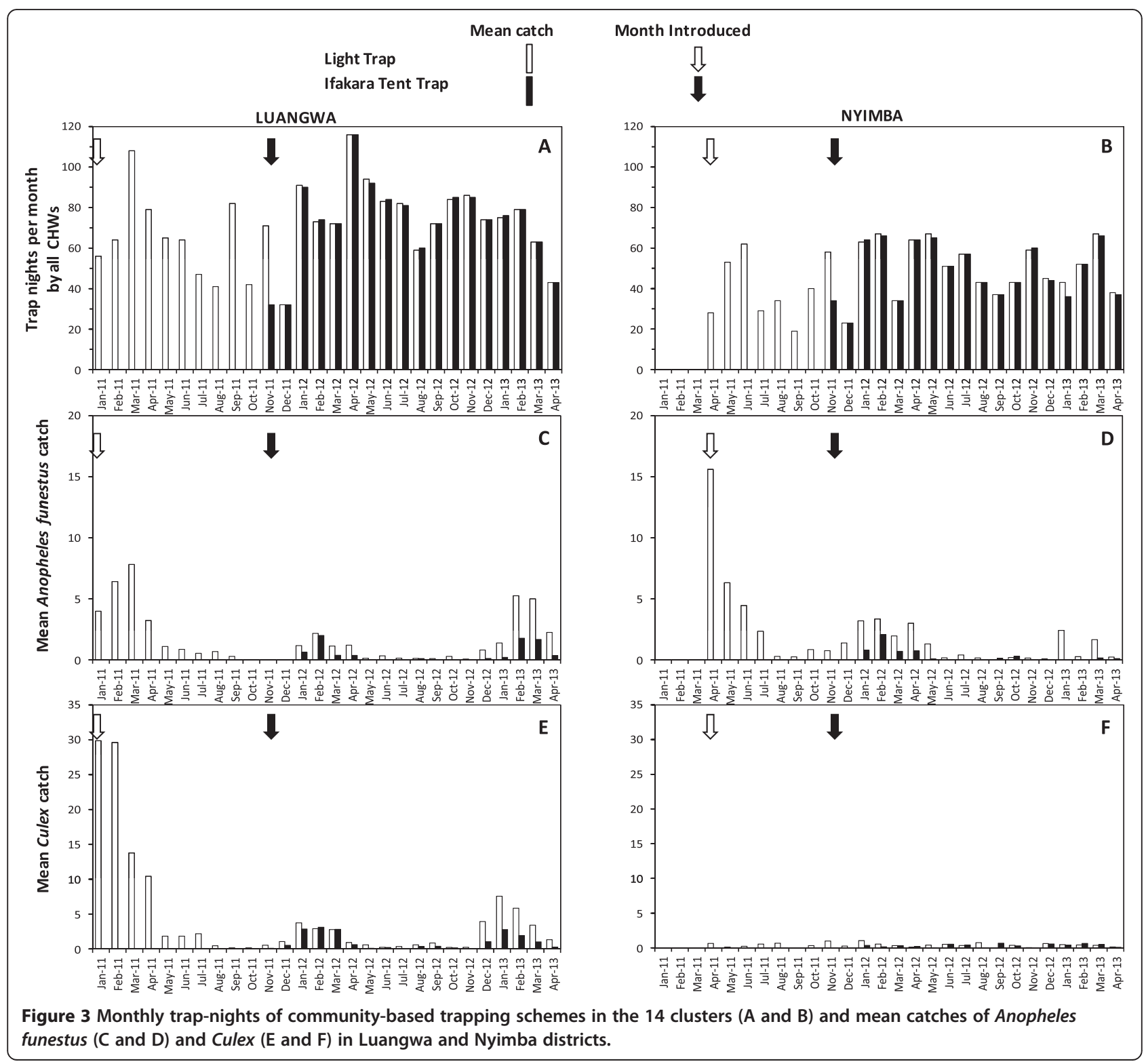


$\$ 15,000$ depreciated to an expected value of $\$ 2,500$ when disposed of by tender after five years of use) as well as the daily remuneration of the CHWs at the rate of ZMW100 (\$19.1) per night of execution of the QA exercise. Whenever QA was conducted in clusters in Nyimba district, accommodation costs were also paid for the CHWs in the QA team because it was impractical for the team to return to their home in Chisobe village (Luangwa) on a daily basis, due to the long distance between the districts and the bad terrain between clusters during the rainy season. The CB CHWs received a minimal monthly incentive in form of the monthly remuneration of ZMW350 (\$66.9) agreed upon at the start of the study. In addition to this incentive, the additional costs incurred included provision of field supplies and having their bicycles repaired in order to facilitate their ease of movement and carrying of the traps to the selected households where the trapping surveys took place.

In estimating these costs, the approximated amount of time and efforts spent on each trapping scheme was also factored into the total expenditure to calculate the cost per sampling night, as well as per single specimen of An. funestus collected. Consideration was restricted to An. funestus because it is overwhelmingly the most important vector mediating malaria transmission in this part of Zambia [21,32].

\section{Ethical considerations}

This protocol was approved by the National Ethics Committee based at the University of Zambia (IRB00001131 of

Table 2 Relative sampling sensitivity of community-based trapping scheme using CDC Light Traps and Ifakara Tent Traps to capture mosquitoes compared with quality assured catches when both operated simultaneously as estimated by generalized linear mixed models

\begin{tabular}{|c|c|c|c|c|c|c|}
\hline \multirow[b]{2}{*}{ Trapping method } & \multicolumn{4}{|c|}{ Quality assurance } & \multicolumn{2}{|c|}{ Community-based } \\
\hline & HLC indoor & HLC outdoor & LT & ITT & LT & ITT \\
\hline Person trap-nights & 20 & 20 & 20 & 20 & 82 & 82 \\
\hline Number of houses sampled & 20 & 20 & 20 & 20 & 76 & 76 \\
\hline Number of clusters surveyed & 13 & 13 & 13 & 13 & 13 & 13 \\
\hline Mean trap-nights per surveyed house & 1.0 & 1.0 & 1.0 & 1.0 & 1.1 & 1.1 \\
\hline Mean trap-nights per cluster & 1.5 & 1.5 & 1.5 & 1.5 & 6.3 & 6.3 \\
\hline \multicolumn{7}{|l|}{ Total catch of female mosquitoes } \\
\hline Anopheles funestus & 174 & 149 & 66 & 46 & 126 & 24 \\
\hline Anopheles quadriannulatus & 10 & 2 & 0 & 0 & 63 & 9 \\
\hline Other anophelines & 9 & 26 & 0 & 0 & 36 & 1 \\
\hline Culex species & 426 & 394 & 94 & 82 & 224 & 112 \\
\hline \multicolumn{7}{|l|}{ Mean catch [95\% confidence interval] } \\
\hline \multirow[t]{2}{*}{ Anopheles funestus } & 4.507 & 3.860 & 1.498 & 1.047 & 0.584 & 0.101 \\
\hline & {$[2.115,9.604]$} & {$[1.807,8.244]$} & {$[0.680,3.300]$} & {$[0.468,2.343]$} & {$[0.284,1.201]$} & {$[0.045,0.227]$} \\
\hline \multirow[t]{2}{*}{ Anopheles quadriannulatus } & 0.097 & 0.019 & 0 & 0 & 0.184 & 0.026 \\
\hline & {$[0.025,0.383]$} & {$[0.003,0.127]$} & {$[\mathrm{NE}]$} & {$[\mathrm{NE}]$} & {$[0.086,0.394]$} & {$[0.010,0.070]$} \\
\hline \multirow[t]{2}{*}{ Other anophelines } & 0.005 & 0.014 & 0 & 0 & 0.016 & 0.000 \\
\hline & {$[0.001,0.046]$} & {$[0.002,0.124]$} & {$[\mathrm{NE}]$} & {$[\mathrm{NE}]$} & {$[0.004,0.071]$} & {$[0.000,0.005]$} \\
\hline \multirow[t]{2}{*}{ Culex species } & 11.941 & 11.044 & 1.743 & 1.374 & 0.305 & 0.146 \\
\hline & {$[5.186,27.494]$} & {$[4.795,25.439]$} & {$[0.771,3.943]$} & {$[0.604,3.126]$} & {$[0.145,0.642]$} & {$[0.069,0.312]$} \\
\hline \multicolumn{7}{|c|}{ Relative rate of capture [95\% confidence interval] } \\
\hline \multirow[t]{2}{*}{ Anopheles funestus } & 1.00 & 0.856 & $0.332^{* * *}$ & $0.232^{* * *}$ & $0.130^{* * *}$ & $0.022^{* * *}$ \\
\hline & & {$[0.688,1.065]$} & {$[0.185,0.596]$} & {$[0.127,0.426]$} & {$[0.079,0.212]$} & {$[0.012,0.041]$} \\
\hline \multirow[t]{2}{*}{ Anopheles quadriannulatus } & 1.00 & $0.200^{*}$ & 0 & 0 & 1.885 & 0.266 \\
\hline & & {$[0.042,0.959]$} & {$[\mathrm{NE}]$} & {$[\mathrm{NE}]$} & {$[0.497,7.153]$} & {$[0.061,1.157]$} \\
\hline \multirow[t]{2}{*}{ Other anophelines } & 1.00 & $2.889^{* *}$ & 0 & 0 & 3.215 & 0.085 \\
\hline & & {$[1.343,6.213]$} & {$[\mathrm{NE}]$} & {$[\mathrm{NE}]$} & {$[0.355,29.131]$} & {$[0.004,1.740]$} \\
\hline \multirow[t]{2}{*}{ Culex species } & 1.00 & 0.925 & $0.146^{* * *}$ & $0.115^{* * *}$ & $0.026^{* * *}$ & $0.012^{* * *}$ \\
\hline & & {$[0.807,1.061]$} & {$[0.075,0.283]$} & {$[0.059,0.224]$} & {$[0.014,0.047]$} & {$[0.007,0.023]$} \\
\hline
\end{tabular}

${ }^{*} \mathrm{P}<0.05,{ }^{* *} \mathrm{P}<0.01,{ }^{* * * \mathrm{P}<0.001 .}$ 
I0RG0000774) and the Ethical Review Board of the Liverpool School of Tropical Medicine (09.60). The benefits and risks associated with participating were explained to participants in advance of seeking their consent and assent. Adult participants had all consented to participate in the study, while all the children involved were permitted to do so by either their parents or guardians and provided assent where old enough. QA team members conducting HLC were administered with dapsonepyrimethamine (Deltaprim ${ }^{\odot}$ ), one of the recommended drugs for chemoprophylaxis in Zambia, every week in accordance with the national guidelines.

\section{Results}

\section{Species composition and abundances}

A total of 20,683 female mosquitoes were collected by both the CB and QA sampling schemes in the 3,174 trap nights (Table 1). Morphological identification showed that the An. funestus group and An. gambiae complex comprised $34.5 \%(\mathrm{n}=7,127)$ and $3.3 \%(\mathrm{n}=685)$, respectively, while other anophelines and the culicines mosquitoes comprised 3.2\% $(n=661)$ and 59.0\% $(n=12,210)$, respectively, of the total. Of the 596 specimens that were initially identified as members of the An. funestus group by routine morphology, and then also successfully identified to species by PCR, 96.5\% ( $\mathrm{n}=575)$ were confirmed to be $A n$. funestus sensu stricto, with the remainder being Anopheles rivulorum $(1.8 \%, \mathrm{n}=11)$ and Anopheles leesoni (1.7\%, $\mathrm{n}=10$ ), respectively. Densities of the An. funestus group, as determined by routine morphological classification can therefore be considered quite a reliable representation of An. funestus s.s. as a species. PCR analysis of mosquitoes from the An. gambiae complex confirmed previous observations [21] that most specimens which amplified $(69 \%$ (49/71)) were An. quadriannulatus, so this taxon is referred to as An. quadriannulatus subsequently in this text. All the other anophelines were morphologically identified as Anopheles coustani (34.0\%; $\mathrm{n}=225)$, Anopheles pretoriensis (22.5\%; $\mathrm{n}=149)$, Anopheles rufipes $(19.1 \% ; \mathrm{n}=126)$, Anopheles squamosis $(13.2 \% ; \mathrm{n}=87)$, Anopheles implexus $(11.0 \% ; \mathrm{n}=73)$ and one $(0.2 \%)$ Anopheles maculipalpis.

Of the total 550 An. funestus s.l. that were tested for circumsporozoite ELISA, only 23 An. funestus were detected with Plasmodium falciparum sporozoites in their salivary glands, corresponding to a sporozoite rate of $4.2 \%$. This sporozoite infection prevalence is considerably higher than that previously reported from Chisobe [21], presumably because the period and geographical scope of sampling were far larger and also possibly because levels of insecticide resistance in the area may have increased. The

Table 3 Crude estimates of the costs per sampling scheme per trap-night and per Anopheles funestus caught for the three months when community-based sampling was validated with quality assurance sampling schemes

\begin{tabular}{|c|c|c|c|c|c|c|}
\hline \multirow[t]{2}{*}{ Estimated parameter } & \multirow[t]{2}{*}{ Units } & \multicolumn{3}{|c|}{ Quality assured } & \multicolumn{2}{|c|}{ Community-based } \\
\hline & & QA-HLC & QA-LT & QA-ITT & CB-LT & CB-ITT \\
\hline Number of samples & Person-night & 40 & 20 & 20 & 249 & 243 \\
\hline Numbers caught & $\begin{array}{l}\text { Number of } \\
\text { An. funestus }\end{array}$ & 526 & 41 & 32 & 637 & 156 \\
\hline Mean caught & $\begin{array}{l}\text { Number of } \\
\text { An. funestus per } \\
\text { person-night }\end{array}$ & 13.2 & 2.1 & 1.6 & 2.6 & 0.6 \\
\hline Personnel costs ${ }^{a}$ & $\$(Z M W)$ & $2,180(11,401.4)$ & $1,520(7,949.6)$ & $1,076(5,627.5)$ & $2509.4(13,124.2)$ & $2,939.4(15,373.1)$ \\
\hline Per diem costs ${ }^{\mathrm{b}}$ & $\$(Z M W)$ & $414(2,165.2)$ & $1,243(6,500.9)$ & $1,243(6,500.9)$ & $621(3,247.8)$ & $621(3,247.8)$ \\
\hline Trap depreciation costs & $\$(\mathrm{ZMW})$ & $0(0)$ & $87.5(457.6)$ & 125(653.8) & $87.5(457.6)$ & $125(653.8)$ \\
\hline Transport costs $^{\mathrm{a}}$ & $\$(\mathrm{ZMW})$ & $225(1,176.8)$ & $225(1,176.8)$ & $225(1,176.8)$ & $0(0)$ & $0(0)$ \\
\hline Vehicle maintenance costs ${ }^{c}$ & $\$(\mathrm{ZMW})$ & $212(1,108.8)$ & $211(1,108.8)$ & $212(1,108.8)$ & $71(371.3)$ & $71(371.3)$ \\
\hline Vehicle depreciation cost ${ }^{d}$ & $\$(Z M W)$ & $2,500(13,075)$ & $2,500(13,075)$ & $2,500(13,075)$ & $0(0)$ & $0(0)$ \\
\hline Bicycle repair costs ${ }^{c}$ & $\$(Z M W)$ & $0(0)$ & $0(0)$ & $0(0)$ & $94(491.6)$ & $611(3,195.5)$ \\
\hline Bicycle depreciation costs ${ }^{d}$ & $\$(\mathrm{ZMW})$ & $0(0)$ & $0(0)$ & $0(0)$ & $5(26.2)$ & $5(26.2)$ \\
\hline Total expenditure & $\$(Z M W)$ & $5,531(28,927.1)$ & $5,788(30,268.6)$ & $5,381(28,142.6)$ & $3,388(17,718.7)$ & $4,372(22,867.7)$ \\
\hline Cost per person-night of sampling & $\$(Z M W)$ & $138.3(723.2)$ & $289.4(1,513.4)$ & $269.1(1,407.1)$ & 13.6(71.2) & 18.0(94.1) \\
\hline Cost per specimen of An. funestus caught & $\$(Z M W)$ & $10.5(55)$ & 141.2(738.3) & $168.2(879.5)$ & $5.3(27.8)$ & $28.0(146.6)$ \\
\hline
\end{tabular}

${ }^{a}$ Cost estimates were based on the approximated time and efforts spent on each trapping method.

${ }^{\mathrm{b}}$ Assumptions made on the salaries paid and per diem to the central level teams during their visits.

${ }^{c}$ Estimated cost incurred for maintaining the equipment for transporting or visiting the trapping schemes per location.

${ }^{\mathrm{d}}$ Monthly depreciation costs calculated when both trapping schemes where operational for three months.

\$- US dollar.

ZMW - Zambian Kwacha.

Note: $1 \$ \approx$ ZMW 5.23 which was the average exchange during the midpoint year of 2012 . 
abundance of An. funestus s.s. reported here across both districts is approximately consistent with previous studies at one of the clusters in Chisobe [21,32] and confirms that it is the predominant species sustaining malaria transmission in this part of Zambia.

\section{Sampling intensity and total catches of community-based trapping}

There was some inconsistency in the number of trap-nights of sampling by the CB trapping schemes over the 28 months of mosquito collections in all the clusters in both districts and the scheduled target sampling intensity was only occasionally achieved in Luangwa and never in Nyimba (Figure 3). It was only in February 2011 and April 2012 when trapnights in Luangwa district exceeded the average of 150 trapnights that had been expected to be attained per month per cluster. Nevertheless, adequate sampling to measure mean mosquito densities was sustained throughout the study. Interestingly, it appears that more trap-nights were conducted during the wet seasons when the CHWs observed increased abundance of An. funestus and Culex species (Figure 3). The overall numbers of person trap-nights conducted by the $\mathrm{CB}$ surveys were $>100$ greater than the QA surveys (Table 1), not only because the former had far greater numbers of staff operating, each of whom sampled with slightly greater frequency, but also because these were conducted over a much longer period of 28 months while the QA were restricted to the last three months of the study.

\section{Comparison of community-based and quality assurance mosquito trapping surveys}

Summaries of the mean number of trap-nights of sampling per household and per cluster surveyed, mean catches and relative rates of capture for each taxon in times and places when both the $\mathrm{CB}$ and $\mathrm{QA}$ surveys were operational are shown in Table 2. The total numbers of person trap-nights and mean number of trap-nights completed per sampled cluster by the $\mathrm{CB}$ scheme were far higher (Table 2), despite the fact that inclusion of this data was restricted to within a week before or after a QA survey in the same cluster, simply because the frequency of sampling with a single, centralized QA team was limited by the practical logistical limitations described in background.

For An. funestus, relative rate of capture per trap-night of the CB-LT was only $13 \%$ when compared with the indoor HLC, while that of CB-ITT was $<3 \%$ (Table 2). However, comparing the CB-LT and the CB-ITT sampling methods with their application through the QA scheme, their relative capture rate per night of trapping was estimated to be $50 \%$ (relative rate $(\mathrm{RR})[95 \%$ confidence interval $(\mathrm{CI})]=0.500$ $[0.299,0.838] ; \mathrm{P}=0.009)$ and $7 \%(\mathrm{RR}[95 \% \mathrm{CI}]=0.069$ $[0.027,0.174] ; \mathrm{P}<0.001)$, respectively. Combined QA surveys with LT and ITT neither captured any Anopheles quadriannulatus nor any other anophelines in the three months these were conducted over. The CB-LT captured more An. quadriannulatus than any other method, including QA-HLC, but overall numbers of this mosquito were so low that this difference was not significant (Table 2). Overall, CB trapping with either LT or ITT exhibited relatively low rates of capture compared with QA surveys of HLC and even with the same trapping methods when conducted simultaneously (Table 2).

Using the mean An. funestus trap catches $\left(\mathrm{M}_{\mathrm{t}}\right)$ by $\mathrm{CB}$ application of LT and ITT, as well as their relative capture rates compared with indoor $\operatorname{HLC}\left(\lambda_{t}\right)$, as estimated by GLMM (Table 2) and the sporozoite prevalence

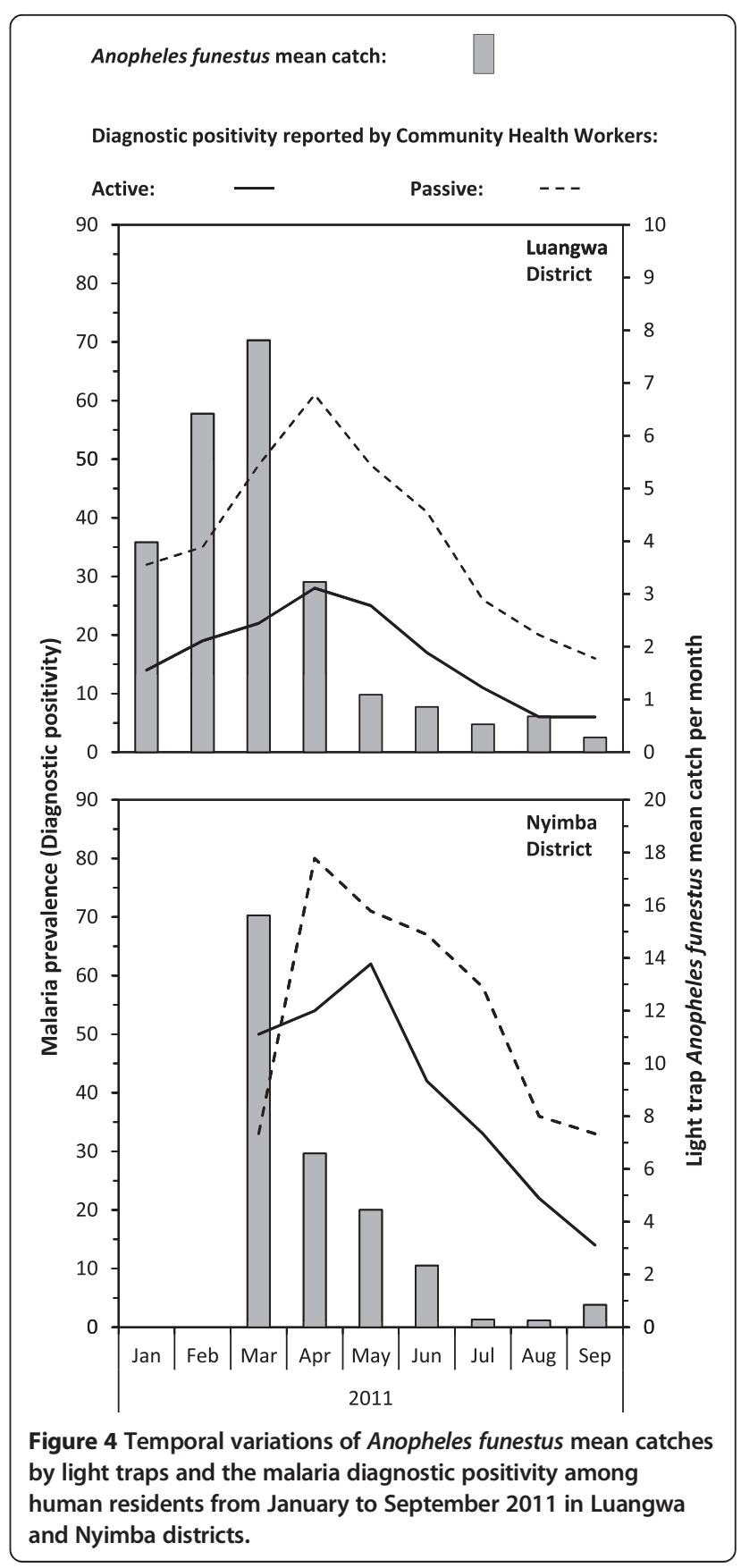


estimate (S) described in the second paragraph of the results section, entomologic inoculation rates $\left(E I R_{t}\right)$ for each of the two traps of 68.6 and 70.1 infectious bites per unprotected per user were calculated $\left(E \mathrm{ER}_{\mathrm{t}}=\mathrm{M}_{\mathrm{t}} \times\right.$ $\left.\mathrm{S} \times 365 / \lambda_{\mathrm{t}}\right)$ assuming that the vast bulk of exposure of unprotected humans occurs indoors in this setting [32].

\section{Cost effectiveness of community-based and quality} assurance surveys for capturing Anopheles funestus Results for the QA-HLC placed indoors and outdoors were combined and considered as a single trapping method. Cost per sampling night was lowest for CB-LT, followed by CB-ITT, which was about twice as expensive, and then far more distantly by the QA survey, which were all at least an order of magnitude more expensive than either $C B$ approach (Table 3). Cost per specimen of An. funestus captured was by far the lowest for CB-LT, followed by the potentially hazardous QA-HLC and then CB-ITT which were approximately five and seven times less cost effective, respectively, and then QA-LT and QA-ITT which were both an order of magnitude less cost effective than either CB method or QA-HLC (Table 3).

\section{Epidemiological relevance of community-based surveys of Anopheles funestus}

Figure 4 shows how the time-trends of malaria parasitaemia over the course of this period approximately follow those for the mean An. funestus catch by the CB entomological surveys. Consistent with previous studies in the area [33] parasite rates were generally much lower in Luangwa than in Nyimba district, with the least transmission recorded in the southernmost corner of the study area, at or near the district capital in Luangwa Boma, and these spatial trends in malaria parasitaemia were clearly associated with An. funestus density (Figure 5).

\section{Discussion}

The CB trapping scheme proved to be far more practical, effective and cost effective for trapping large numbers of An. funestus because the higher frequency and overall numbers of mosquito samples collected within each population cluster captures temporal trends with far greater resolution and precision than conventional surveys by centralized teams, as exemplified by the QA surveys described herein. Familiarity of the CHWs with the communities and the collection sites enabled convenient, repeated, high frequency trapping in each cluster, simply because the CHWs live where they work. Overall, CB trapping with either LT or ITT exhibited relative low rates of capture per night of sampling compared with HLC, or even with the same trapping methods, implemented by the QA team. Nevertheless, $\mathrm{CB}$ trapping schemes caught far more mosquito of all taxa simply because these procedures allowed for more intensive sampling of each cluster in terms of trap-nights conducted over the whole period of the study. While longitudinal surveillance $\mathrm{CB}$ trapping scheme may not be as sensitive as the gold standard HLC in terms of estimating the absolute biting densities of host-seeking vectors, such assessments merely reflect the efficiency of the trapping method rather than the effectiveness of the system through which they are applied. When evaluated in terms of cost-effectiveness, it does appear to represent a far more affordable option for routine vector population dynamics monitoring at programmatic level that yields far more spatial and, especially, temporal resolution than is otherwise possible.

The only previous study to have validated the affordability, accuracy and epidemiological relevance of a $\mathrm{CB}$

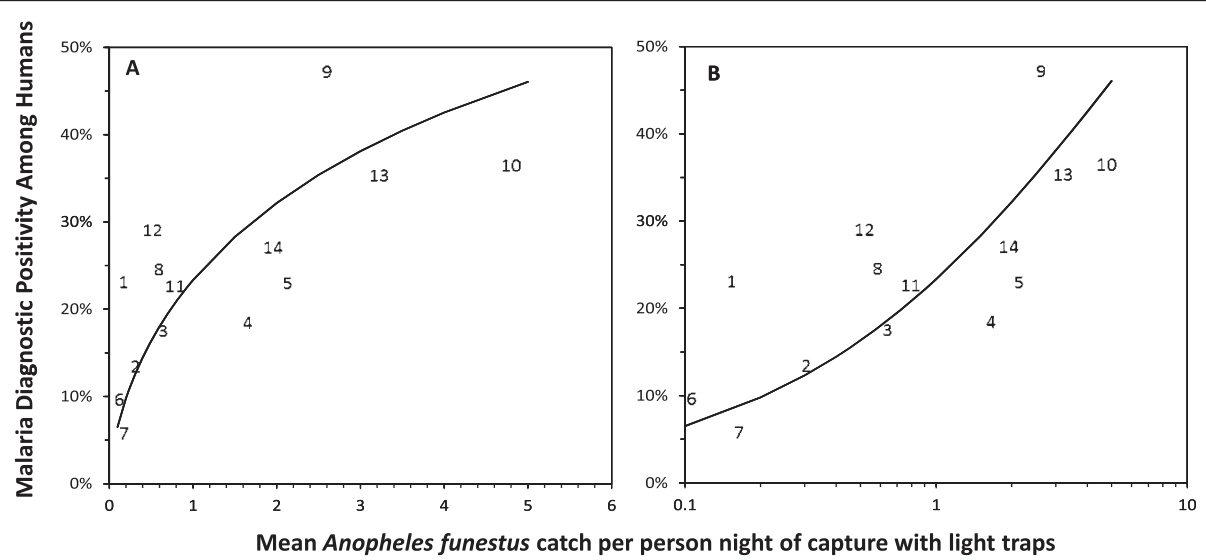

Figure 5 Relationship between malaria diagnostic positivity among human residents and mean catches of Anopheles funestus per trap night of capture with light traps in each cluster, plotted with a standard (A) and logarithmic (B) horizontal axis. Each data point represents the mean diagnostic positivity across all ages for a single cluster, numbered as described in Figure 2 . The plotted lines represent the generalized linear mixed model fitted to these data, as described in the entomological and epidemiological data analysis section. Odds ratio [95\% Confidence interval] for a ten-fold increase in the mean Anopheles funestus catch $=4.378[2.438,7.8580] ; \mathrm{P}<0.001$. 
trapping system relates to a municipal-scale platform for monitoring and evaluating the impact of an urban larviciding programme where An. gambiae s.s. is the predominant vector present [15]. The findings reported here also provide the first evidence of the applicability of quality assured CB trapping schemes in a transmissions system where local vectorial capacity is dominated by An. funestus. Unlike the preceding example from an urban Tanzanian setting, which necessarily relied on the locally designed and effective ITT [15], this study demonstrates for the first time how solar-recharged LT can be practically applied by CB staff to yield vector density data that predict malaria risk infection in 14 clusters distributed across $>14,000 \mathrm{sq} \mathrm{km}$ of an isolated part of rural Zambia (Figures 4 and 5). The sampled clusters were far too widely distributed across these two districts for the QA team to visit more than once or twice every three months and these same logistical limitations are likely to apply to any centralized QA surveillance system with finite human and financial resources, especially if attempting to monitor vector populations on larger provincial or national scales. While others $[34,35]$ have used CB trapping schemes to evaluate large-scale intervention progress, none conducted QA or estimated costs incurred under conditions comparable with programmatic operational conditions. The observations reported here therefore complement these earlier studies and provide another encouraging example of how much can be achieved by imparting basic entomological skills to non-specialist $\mathrm{CB}$ staff and availing them with minimal resources to monitor vector population dynamics and how it responds to control in their own communities.

Like all studies, this evaluation had limitations that merit careful consideration. The QA validation exercise was only carried out for three months during the rainy season so it can only be assumed, rather than proven, that these comparisons are representative of $\mathrm{CHW}$ and trap performance throughout the study. Furthermore, the CHWs were informed approximately one day in advance that the QA team would be coming to visit the cluster so it is possible that they conducted a small proportion of their trapping in that interim period more carefully than they normally would. Future studies of $\mathrm{CB}$ trapping schemes, especially those evaluating prototype systems operating at larger scales, should therefore incorporate continuous, randomized and unannounced, if not necessarily as intensive, QA surveys. It was also observed that the CHWs often conducted lower numbers of trap nights of sampling during the dry season when the catches were lowest because they thought it unnecessary to continue collecting even when the catches were often zero. It may, therefore, be necessary to sensitize $C B$ staff to the critical importance of measuring the low but non-zero vector densities that occur in the dry season, especially in the context of any pre-elimination scenario where supplementary mass drug administration, mass screen and treat, or vector control measures are specifically introduced and evaluated as interventions to achieve termination of local transmission.

\section{Conclusions}

Despite these study limitations, the prototype $\mathrm{CB}$ mosquito trapping scheme evaluated here clearly has considerable potential for improvement and scale-up. It is therefore recommended that future operational studies are undertaken to adapt, optimize and evaluate $\mathrm{CB}$ trapping schemes for monitoring mosquito population dynamics at nationally representative scales so that the influence of physiological and phenotypic traits, as determinants of success, limitations and failures of vector population control, can be assessed continuously, indefinitely and sustainably.

\section{Abbreviations}

CB: Community-based; CHW: Community health worker; CS: Capsule suspension; ELISA: Enzyme-linked immunosorbent assay; ES: Emulsifiable suspension; GMEP: Global Malaria Eradication Programme; HLC: Human landing catch; IRS: Indoor residual spraying; NMCC: National Malaria Control Centre; NMCP: National Malaria Control Programme; IRB: Institutional Review Board; ITT: Ifakara Tent Trap; kdr: Knock down resistance; LT: Centers for Disease Control and Prevention miniature Light Traps; LLIN: Long-lasting insecticidal net; RDT: Rapid diagnostic test; QA: Quality assurance; ZMW: Zambian Kwacha.

\section{Competing interests}

The authors declare that they have no competing interests.

\section{Authors' contributions}

GFK, AS, and CHS conceived and planned the study. CHS, AS, DC, JC, BH, and MK supervised execution of the study. NFL, MM and IM conducted the laboratory analyses. CHS analysed the data and drafted the manuscript in consultation with GFK. All authors contributed to editing of the manuscript. All authors read and approved the final manuscript.

\section{Acknowledgements}

We thank the Ministry of Health (Dr Peter Mwaba and Dr Elizabeth ChizemaKawesha), National Malaria Control Centre, the Zambian Malaria Transmission Consortium project team, the District Health Offices and communities of Luangwa and Nyimba for their co-operation and support. We thank Dr Adam Bennett for providing the map for the study site. We thank Dr Nicodem Govella for arranging supply of ITT-C and Dr Kate Aultman of the Bill \& Melinda Gates Foundation for suggesting evaluation of mosquito trapping systems in terms of their epidemiological predictive power. Financial support was provided by the Bill \& Melinda Gates Foundation through the Malaria Transmission Consortium (Award number 45114).

\section{Author details}

'National Malaria Control Centre, Chainama Hospital College Grounds, Off Great East road, P.O. Box 32509 Lusaka, Zambia. ${ }^{2}$ Vector Biology Department, Liverpool School of Tropical Medicine, Pembroke Place, Liverpool L3 5QA, UK. ${ }^{3}$ Department of Biological Sciences, University of Notre Dame, Eck Institute for Global Health, Notre Dame, IN 46556, USA. ${ }^{\text {IIfakara Health }}$ Institute, Environmental Health and Ecological Sciences Thematic Group, PO Box 53, Ifakara, Morogoro, United Republic of Tanzania.

Received: 24 January 2014 Accepted: 21 May 2014

Published: 7 June 2014

\section{References}

1. Moonen B, Cohen JM, Snow RW, Slutsker L, Drakeley C, Smith DL, Abeyasinghe RR, Rodriguez MH, Maharaj R, Tanner M, Targett G: 
Operational strategies to achieve and maintain malaria elimination. Lancet 2010, 376:1592-1603.

2. Killeen GF, Seyoum A, Sikaala C, Zomboko AS, Gimnig JE, Govella NJ, White MT: Eliminating malaria vectors. Parasit Vectors 2013, 6:172

3. Smith DL, Cohen JM, Chiyaka C, Johnston G, Gething PW, Gosling R, Buckee CO, Laxminarayan R, Hay SI, Tatem AJ: A sticky situation: the unexpected stability of malaria elimination. Philos Trans R Soc Lond B Biol Sci 2013, 368:20120145.

4. Durnez L, Coosemans M: Residual Transmission of Malaria: An Old Issue for New Approaches. InTech: Antwerp; 2013.

5. Ferguson HM, Dornhaus A, Beeche A, Borgemeister C, Gottlieb M, Mulla MS, Gimnig JE, Fish D, Killeen GF: Ecology: a prerequisite for malaria elimination and eradication. PLoS Med 2010, 7:e1000303.

6. Govella NJ, Chaki PP, Killeen GF: Entomological surveillance of behavioural resilience and resistance in residual malaria vector populations. Malar $J$ 2013, 12:124.

7. Killeen GF: A second chance to tackle African malaria vector mosquitoes that avoid houses and don't take drugs. Am J Trop Med Hyg 2013, 88:809-816.

8. Russell TL, Beebe NW, Cooper RD, Lobo NF, Burkot TR: Successful malaria elimination strategies require interventions that target changing vector behaviours. Malar J 2013, 12:56.

9. Griffin JT, Hollingsworth TD, Okell LC, Churcher TS, White M, Hinsley W, Bousema T, Drakeley CJ, Ferguson NM, Basáñez MG, Ghani AC: Strategies towards Plasmodium falciparum malaria elimination in Africa using currently available tools. PLOS Med 2010, 7:e1000324

10. Eckhoff PA: Mathematical models of within-host and transmission dynamics to determine effects of malaria Interventions in a variety of transmission settings. Am J Trop Med Hyg 2013, 88:817-827.

11. Killeen GF, Chitnis N: Potential causes and consequences of behavioural resilience and resistance in malaria vector populations: a mathematical modelling analysis. Malar J 2014, 13:97.

12. Cohen JM, Smith DL, Cotter C, Ward A, Yamey G, Sabot OJ, Moonen B: Malaria resurgence: a systematic review and assessment of its causes. Malar J 2012, 11:122

13. Gatton ML, Chitnis N, Churcher T, Donnelly MJ, Ghani AC, Godfray HC, Gould F, Hastings I, Marshall J, Ranson H, Rowland M, Shaman J, Lindsay SW: The importance of mosquito behavioural adaptations to malaria control in Africa. Evolution 2013, 67:1218-1230.

14. Mukabana WR, Kannady K, Kiama GM, ljumba JN, Mathenge EM, Kiche I, Nkwengulila G, Mboera L, Mtasiwa D, Yamagata Y, van Schayk I, Knols BG, Lindsay SW, Caldas De Castro M, Mshinda H, Tanner M, Fillinger U, Killeen GF: Ecologists can enable communities to implement malaria vector control in Africa. Malar J 2006, 5:9.

15. Chaki PP, Mlacha Y, Msellemu D, Muhili A, Malishee AD, Mtema ZJ, Kiware SS, Zhou Y, Lobo NF, Russell TL, Dongus S, Govella NJ, Killeen GF: An affordable, quality-assured community-based system for high-resolution entomological surveillance of vector mosquitoes that reflects human malaria infection risk patterns. Malar J 2012, 11:172

16. Killeen GF, Tanner M, Mukabana WR, Kalongolela MS, Kannady K, Lindsay SW, Fillinger U, de Castro MC: Habitat targeting for controlling aquatic stages of malaria vectors in Africa. Am J Trop Med Hyg 2006, 74:519-520.

17. Govella NJ, Chaki PP, Geissbuhler Y, Kannady K, Okumu F, Charlwood JD, Anderson RA, Killeen GF: A new tent trap for sampling exophagic and endophagic members of the Anopheles gambiae complex. Malar J 2009, 8:157.

18. Govella NJ, Moore JD, Killeen GF: An exposure-free tool for monitoring adult malaria mosquito populations. Am J Trop Med Hyg 2010, 83:596-600.

19. Govella NJ, Chaki PP, Mpangile JM, Killeen GF: Monitoring mosquitoes in urban Dar es Salaam: evaluation of resting boxes, window exit traps, CDC light traps, Ifakara tent traps and human landing catches. Parasit Vectors 2011, 4:40.

20. National Malaria Control Centre: National Malaria Control Programme Strategic Plan for FY 2011-2015: Consolidating Malaria Gains for Impact. Lusaka, Zambia: Ministry of Health; 2012.

21. Sikaala CH, Killeen GF, Chanda J, Chinula D, Miller JM, Russell TL, Seyoum A: Evaluation of alternative mosquito sampling methods for malaria vectors in Lowland South-East Zambia. Parasit Vectors 2013, 6:91.

22. Hamainza B, Hawela M, Sikaala CH, Kamuliwo M, Bennett A, Eisele TP, Mille J, Seyoum A, Killeen GF: Monitoring, characterization and control of chronic, symptomatic malaria infections in rural Zambia through monthly household visits by paid community health workers. Malar J 2014, 13:128.
23. $\mathrm{MOH}$ : Zambia National Indicator Survey report. Lusaka, Zambia: Ministry of Health; 2013.

24. $\mathrm{MOH}$ : Zambia National Indicator Survey report. Lusaka, Zambia: Ministry of Health; 2010.

25. Chanda E, Hemingway J, Kleinschmidt I, Rehman AM, Ramdeen V, Phiri FN, Coetzer S, Mthembu D, Shinondo CJ, Chizema-Kawesha E, Kamuliwo M, Mukonka V, Baboo KS, Coleman M: Insecticide resistance and the future of malaria control in Zambia. PLOS One 2011, 6:e24336.

26. Gillies MT, Coetzee M: Supplement to the Anophelinae of Africa South of the Sahara (Afrotropical Region). Johannesburg: South African Medical Research Institute; 1987.

27. Silver JB, Service MW: Mosquito Ecology: Field Sampling Methods. London: Springer; 2008.

28. Burkot TR, Williams JL, Schneider I: Identification of Plasmodium falciparum-infected mosquitoes by a double antibody enzyme-linked immunosorbent assay. Am J Trop Med Hyg 1984, 33:783-788.

29. Durnez L, Van Bortel W, Denis L, Roelants P, Veracx A, Trung HD, Sochantha T, Coosemans M: False positive circumsporozoite protein ELISA: a challenge for the estimation of the entomological inoculation rate of malaria and for vector incrimination. Malar J 2011, 10:195.

30. Scott JA, Brogdon WG, Collins FH: Identification of single specimens of the Anopheles gambiae complex by the polymerase chain reaction. Am J Trop Med Hyg 1993, 49:520-529.

31. Koekemoer LL, Lochouarn L, Hunt RH, Coetzee M: Single-strand conformation polymorphism analysis for identification of four members of the Anopheles funestus (Diptera: Culicidae) group. J Med Entomol 1999, 36:125-130.

32. Seyoum A, Sikaala $\mathrm{CH}$, Chanda J, Chinula D, Ntamatungiro AJ, Hawela M, Miller $J M$, Russell TL, Briet OJ, Killeen GF: Human exposure to anopheline mosquitoes occurs primarily indoors, even for users of insecticide-treated nets in Luangwa Valley, South-east Zambia. Parasit Vectors 2012, 5:101.

33. Keating J, Miller JM, Bennet A, Moonga HB, Eisele TP: Plasmodium falciparum parasite infection prevalence from a household survey in Zambia using microscopy and a rapid diagnostic test: implications for monitoring and evaluation. Acta Trop 2009, 112:277-282.

34. Mouatcho JC, Hargreaves K, Koekemoer LL, Brooke BD, Oliver SV, Hunt RH, Coetzee M: Indoor collections of the Anopheles funestus group (Diptera: Culicidae) in sprayed houses in northern KwaZulu-Natal, South Africa. Malar J 2007, 6:30

35. Sharp BL, Ridl FC, Govender D, Kuklinski J, Kleinschmidt I: Malaria vector control by indoor residual insecticide spraying on the tropical island of Bioko, Equatorial Guinea. Malar J 2007, 6:52.

doi:10.1186/1475-2875-13-225

Cite this article as: Sikaala et al:: A cost-effective, community-based, mosquito-trapping scheme that captures spatial and temporal heterogeneities of malaria transmission in rural Zambia. Malaria Journal 2014 13:225.

\section{Submit your next manuscript to BioMed Central and take full advantage of:}

- Convenient online submission

- Thorough peer review

- No space constraints or color figure charges

- Immediate publication on acceptance

- Inclusion in PubMed, CAS, Scopus and Google Scholar

- Research which is freely available for redistribution 